\title{
Variational Monte Carlo method for the Baeriswyl wave function: Application to the one-dimensional bosonic Hubbard model
}

\author{
B. Hetényi, ${ }^{1}$ B. Tanatar, ${ }^{1}$ and L. M. Martelo ${ }^{2,3}$ \\ ${ }^{1}$ Department of Physics, Bilkent University, TR-06800 Bilkent, Ankara, Turkey \\ ${ }^{2}$ Departamento de Engenharia Física, Faculdade de Engenharia da Universidade do Porto, Rua Dr. Roberto Frias, 4200-465 Porto, Portugal \\ ${ }^{3}$ Centro de Física do Porto, Faculdade de Ciências da Universidade do Porto, Rua do Campo Alegre, 687, 4169-007 Porto, Portugal
}

(Received 8 December 2014; revised manuscript received 4 May 2016; published 25 May 2016)

\begin{abstract}
A variational Monte Carlo method for bosonic lattice models is introduced. The method is based on the Baeriswyl projected wave function. The Baeriswyl wave function consists of a kinetic energy based projection applied to the wave function at infinite interaction, and is related to the shadow wave function already used in the study of continuous models of bosons. The wave function at infinite interaction, and the projector, are represented in coordinate space, leading to an expression for expectation values which can be evaluated via Monte Carlo sampling. We calculate the phase diagram and other properties of the bosonic Hubbard model. The calculated phase diagram is in excellent agreement with known quantum Monte Carlo results. We also analyze correlation functions.
\end{abstract}

DOI: 10.1103/PhysRevB.93.174518

\section{INTRODUCTION}

Variational Monte Carlo is a powerful tool to calculate the properties of quantum systems. In general, expectation values of physical quantities over conveniently chosen variational wave functions allow the application of Monte Carlo sampling methods. For fermionic lattice models, commonly used variational wave functions are the Gutzwiller [1,2] and Baeriswyl [3-5] wave functions (GWF and BWF, respectively). The GWF starts with a noninteracting wave function, and projects out configurations according to the interaction. For fermionic systems, evaluation of physical quantities can be done approximately via a combinatorial approximation, or exactly in the case of the one-dimensional [6,7] and the infinite [7-9] dimensional case. In between those two cases the state-of-the-art is the Monte Carlo method developed by Yokoyama and Shiba [10,11]. For bosonic systems, the GWF reduces to mean-field theory [12].

The BWF can be considered the counterpart of the GWF: the starting point is the wave function with infinite interaction, and the projection applied thereonto is a function of the hopping energy. For fermionic systems this wave function already has a history $[3-5,13,14]$. For a model of interacting spinless fermions the BWF produces excellent results for the ground state energy [14]. We note also, that a method known as the momentum dependent local ansatz, in which momentum dependent amplitudes of pairs are used as variational parameters, was recently developed [15-17]. While there are a number of schemes to solve the BWF for fermionic systems, it has, to the best of our knowledge, not been applied to bosonic systems.

In this work we develop a variational Monte Carlo (VMC) method for correlated bosonic models based on the BWF and apply it to the bosonic Hubbard model (BHM) [18,19] with on-site interaction. The BHM was originally proposed to study actual materials (bosons in porous materials), but they were recently also realized experimentally as ultracold gases in optical lattices [20,21]. The BHM has been treated by analytical and numerical means, including mean-field theory [12,19], perturbative expansion [22], quantum Monte Carlo (QMC) [23-28], density matrix renormalization group
(DMRG) [29-33], and exact diagonalization (ED) [34]. DMRG is limited to one dimension, QMC is limited to small system sizes, and ED is limited to even smaller system sizes. Our variational Monte Carlo approach is shown to give good quantitative results, at the same time, it is not restricted to one dimension, and is less computationally demanding than QMC or ED. It can also be generalized to more complex bosonic strongly correlated models with distance dependent interaction, and/or disorder.

We calculate some of the properties of the BHM. As expected, the ground state energy obtained using our VMC method has a lower value than the one given by mean-field theory. More importantly, for the phase diagram, our results are in excellent quantitative agreement with the quantum Monte Carlo results of Rousseau et al. [26,35,36]. We also obtain the Kosterlitz-Thouless point at the tip of the Mott lobes, and find that our calculations underestimate the values calculated by others [27-31,34]. We also calculate the one-particle reduced density matrix at integer and away from integer fillings. For integer fillings we find decay to zero. The decay is well approximated by an exponential function, implying the absence of a condensate.

The rest of this paper is organized as follows. In the following section we describe in detail the variational Monte Carlo method and our implementation of it for the BHM. In Sec. III we present our results for the phase diagram and one-body density matrix. Subsequently, we conclude our work.

\section{MODEL AND METHOD}

\section{A. Bosonic Hubbard model and the Baeriswyl variational wave function}

We study the BHM with nearest neighbor hopping in one dimension at fixed particle number. The Hamiltonian is

$$
H=-J \sum_{x=1}^{L}\left(\hat{c}_{x+1}^{\dagger} \hat{c}_{x}+\hat{c}_{x}^{\dagger} \hat{c}_{x+1}\right)+U \sum_{x=1}^{L} \hat{n}_{x}\left(\hat{n}_{x}-1\right),
$$

where $L$ denotes the number of sites, and $J$ and $U$ are the hopping and interaction parameters, respectively. The BWF 
has the form

$$
\left|\Psi_{B}\right\rangle=\exp (-\alpha \hat{T})\left|\Psi_{\infty}\right\rangle,
$$

where $\alpha$ denotes the variational parameter, and $\left|\Psi_{\infty}\right\rangle$ is the wave function at $U=\infty . \hat{T}$ denotes the hopping operator [first term in Eq. (1)]. The idea of the Baeriswyl wave function is to start with the infinitely interacting wave function, and act on it with a projector which implements hoppings.

\section{B. Variational Monte Carlo}

The expectation value of an operator $\hat{O}$ can be written as

$$
\langle\hat{O}\rangle=\left\langle\Psi_{B}|\hat{O}| \Psi_{B}\right\rangle=\frac{\left\langle\Psi_{\infty}\left|e^{-\alpha \hat{T}} \hat{O} e^{-\alpha \hat{T}}\right| \Psi_{\infty}\right\rangle}{\left\langle\Psi_{\infty}\left|e^{-2 \alpha \hat{T}}\right| \Psi_{\infty}\right\rangle} .
$$

The following derivations will treat a one particle system, but they are generally applicable. We also assume that the operator $\hat{O}$ is diagonal in the coordinate representation. Inserting coordinate identities $\sum_{x}|x\rangle\langle x|=1$ results in

$$
\langle\hat{O}\rangle=\sum_{x_{L}} \sum_{x_{C}} \sum_{x_{R}} P\left(x_{L}, x_{C}, x_{R}\right) O\left(x_{C}\right),
$$

where the probability distribution $P\left(x_{L}, x_{C}, x_{R}\right)$ is

$$
P\left(x_{L}, x_{C}, x_{R}\right)=\frac{1}{\Omega}\left\langle\Psi_{\infty} \mid x_{L}\right\rangle K\left(x_{L}, x_{C}\right) K\left(x_{C}, x_{R}\right)\left\langle x_{R} \mid \Psi_{\infty}\right\rangle,
$$

where

$$
K\left(x, x^{\prime}\right)=\left\langle x|\exp (-\alpha \hat{T})| x^{\prime}\right\rangle,
$$

with $\Omega$ the normalization determined by the requirement that

$$
\sum_{x_{L}, x_{C}, x_{R}} P\left(x_{L}, x_{C}, x_{R}\right)=1 \text {. }
$$

The quantum particle is represented by three coordinates which we call the "left," "center," and "right" coordinates. Operators diagonal in the coordinate representation can be evaluated using the center coordinate. In quantum Monte Carlo based methods, whether finite temperature [37,38] or ground state [39-41], each particle is represented by a large number of coordinates (Trotter slices) whose number must be increased for accurate results as the temperature is lowered. Therefore, while our method is not exact as the QMC is, it is significantly less demanding of computational resources. Within our VMC method we can reach larger system sizes. In this work we limit ourselves to system sizes with $L=50,100$, in order to have a direct reliable comparison to the available QMC results.

The kinetic energy projection operator can be expressed as

$$
K\left(x, x^{\prime}\right)=\frac{1}{L} \sum_{k} \exp \left[-\alpha \epsilon_{k}+i k\left(x-x^{\prime}\right)\right],
$$

and is shown in Fig. 1. As $\alpha$ increases the propagator also increases in value, allowing for delocalization through increased quantum fluctuations. Given that $P\left(x_{L}, x_{C}, x_{R}\right)$ is positive and normalized, MC techniques can be applied to evaluate expectation values. In the continuum limit, the kinetic energy propagator reads $K(r)=I_{r}(2 \alpha J)$, where $I_{n}(x)$ are the modified Bessel functions of the first kind.

The kinetic energy can be evaluated by constructing an estimator based on taking the logarithmic derivative of the

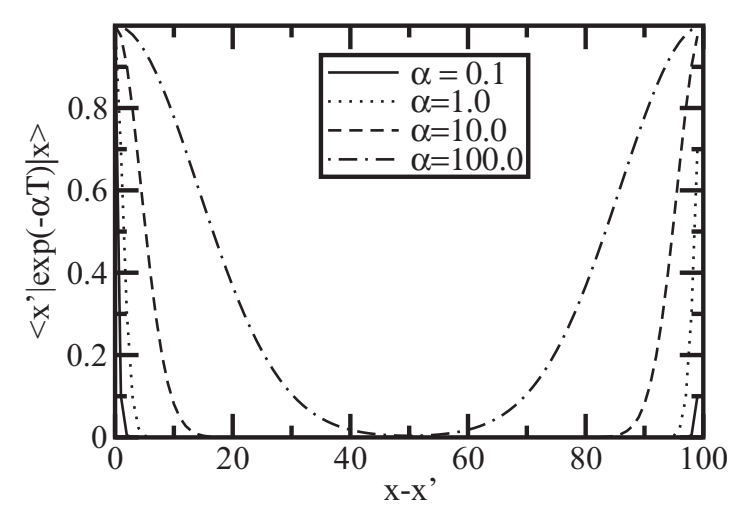

FIG. 1. Kinetic energy propagator as a function of distance for different values of $\alpha$.

quantity $\Omega$ with respect to the variational parameter $\alpha$. We can write the normalization as

$$
\Omega=\left\langle\Psi_{\infty}|\exp (-2 \alpha \hat{T})| \Psi_{\infty}\right\rangle,
$$

and the average kinetic energy as

$$
\langle T\rangle=-\frac{1}{2 \Omega} \frac{\partial \Omega}{\partial \alpha} .
$$

Writing $\Omega$ in terms of the projected wave function one can show that

$$
\langle T\rangle=\sum_{x_{L}, x_{C}, x_{R}} P\left(x_{L}, x_{C}, x_{R}\right) T\left(x_{L}, x_{C}, x_{R}\right),
$$

where

$$
T\left(x_{L}, x_{C}, x_{R}\right)=-\frac{1}{2}\left[\frac{\partial \ln K\left(x_{L}, x_{C}\right)}{\partial \alpha}+\frac{\partial \ln K\left(x_{C}, x_{R}\right)}{\partial \alpha}\right] \text {. }
$$

The generalization to the many-body case is straightforward, but it is in order to make some comments. A typical configuration is represented in Fig. 2. In that figure a lattice of six sites is shown. The left, center, and right replicas of the lattice are all represented. A single quantum particle is

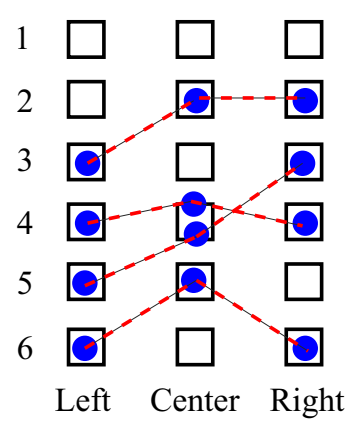

FIG. 2. Representation of our variational algorithm. In our method there are three replicas of the system, labeled left, center, and right. The black squares represent the lattice sites of the one-dimensional system for each such replica. The blue filled circles represent particles. Each particle is represented by one replica on the left, center, and right lattices. The dashed red line represents the kinetic energy projection operator [Eq. (8)]. The left and right replicas correspond to the infinitely interacting system. Since in the case there would be an infinite energy cost, there are no sites with more than one particle among the left and right replicas. In the center replica of the lattice, two or more particles can be on the same lattice. 
represented by three classical particles, one on each lattice replica. The dashed lines in the figure connecting the three particles refer to the kinetic energy projector $K\left(x, x^{\prime}\right)$ [Eq. (8)]. Since the left and right coordinates refer to the infinite interaction wave function $\left(\Psi_{\infty}\right)$ no configuration occurs in which more than one particle is on a particular lattice site. This will be the case for fillings less than one. In general for a filling of $n$ the left and right replicas will only have lattice sites with $\operatorname{int}(n)$ or $\operatorname{int}(n)+1$ particles. However, in the center replica, the lattice sites with any number of particles can occur, since the projector does not place any restrictions there. Since the casting of our method above is in terms of first quantization, exchange is implemented by explicit exchange moves of pairs of particles on the left or right lattices. One randomly chooses a pair and then propose the exchange as a Monte Carlo move. This is similar to how it is done in the continuous quantum Monte Carlo methods, such as path-integral Monte Carlo [38].

\section{One-particle reduced density matrix}

A quantity of general interest is the one-particle reduced density matrix (RDM). The RDM gives information about Bose-Einstein condensation [42]: if it tends to a finite value at long distance, a condensate is present in the system. The RDM (in our case for the BWF) is given by

$$
\rho(y, x)=\left\langle\Psi_{B}\left|\hat{c}_{y}^{\dagger} \hat{c}_{x}\right| \Psi_{B}\right\rangle .
$$

The difficulty with calculating this quantity stems from the fact that it is not diagonal in the coordinate representation. While this is also true for the kinetic energy, there only nearestneighbor hoppings contribute, moreover, one can simply take the derivative with respect to the variational parameter.

In the context of our variational method, the operator $\hat{c}_{x}^{\dagger} \hat{c}_{y}$ corresponds to a virtual hopping from $y$ to $x$, and has the effect of giving rise to virtual configurations in which a given particle has two central coordinates. One of these is located at $x$, the other at $y$. One of these $(x)$ is connected to the left coordinate of the given particle via a Baeriswyl projector, the other $(y)$ to the right coordinate. To calculate $\rho(x, y)$ one starts with a regular configuration, obtained from the Monte Carlo sampling outlined above. One chooses a particle (say, with coordinates $x_{L}, x, x_{R}$, with $x$ denoting the central coordinate) and calculates the ratio

$$
\gamma=\frac{K\left(x_{L}, y\right)}{K\left(x_{L}, x\right)} .
$$

Part of $\rho(x, y)$ is the average of contributions of this type. The scenario for calculating such contributions to the RDM is visually represented in Fig. 3. The two Baeriswyl projectors in the expression in Eq. (14) are shown in the figure by the solid straight lines. For integer fillings, averaging over configurations of this type is all that is needed.

In general, there is another class of virtual configurations which needs to be considered. Away from integer filling, there are holes among the left and right coordinates. As such, the virtual hopping to which $\hat{c}_{x}^{\dagger} \hat{c}_{y}$ corresponds can also move either the left or the right coordinate. In Fig. 4 this state of affairs is represented. In this case the quantity which must be considered is

$$
\gamma=\frac{K\left(y_{L}, y\right)}{K\left(x_{L}, x\right)},
$$

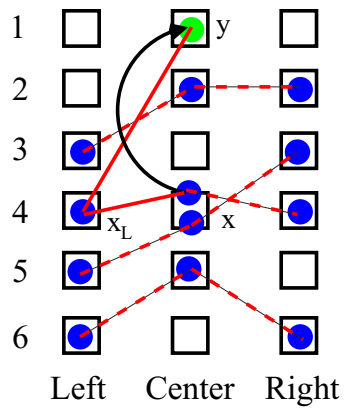

FIG. 3. Representation of a configuration with virtual hopping which contributes to the one-particle reduced density matrix [Eq. (13)]. The virtual hopping takes place from site $y$ to site $x$, indicated by the black semicircular arrow. The contribution to the average reduced density matrix is the proportion of the Baeriswyl propagators denoted by the straight solid red lines [see also Eq. (14)]. The propagators connect the left coordinate of the particle considered (in this case site 4) to the central coordinate after virtual hopping (in this case site 1, particle represented by green circle), and the propagator between the left coordinate to the unmoved central coordinate of the particle (site 4 , particle represented by blue circle).

where $y_{L}$ represents the site to which $x_{L}$ is moved. In the original configuration, from which this virtual configuration is sampled, this site is an empty site [or for fillings $n>1$, they are sites with $\operatorname{int}(n)$ number of particles, rather than int $(n)+1]$.

\section{Relation to shadow wave function}

The BWF is the lattice analog of the shadow wave function (SWF) $[43,44]$, used often in continuous systems in the study of supersolidity. To show this we consider a one-dimensional

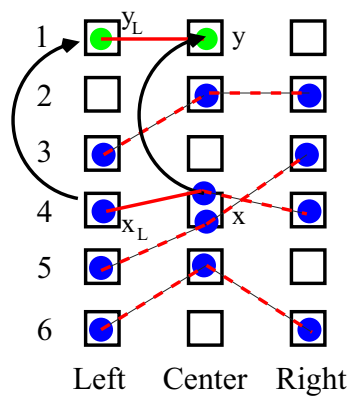

FIG. 4. Representation of a configuration with virtual hopping which contributes to the one-particle reduced density matrix [Eq. (13)]. The virtual hopping takes place from site $y$ to site $x$, indicated by the black semicircular arrow. At the same time, a virtual hopping takes place among the left coordinates, also indicated by a black semicircular arrow. The contribution to the average reduced density matrix is the proportion of the Baeriswyl propagators denoted by the straight solid red lines [see also Eq. (15)]. The propagators connect the left coordinate of the particle (site 1, particle represented by green circle) to the central coordinate of the particle (site 1, particle represented by green circle), both after the virtual hopping, and the propagator left coordinate (site 4 , represented by a blue circle) and the central coordinate of the particle (site 4 , represented by a blue circle) both before the virtual hopping. 
system with Hamiltonian

$$
H=-\frac{\hbar^{2}}{2 m} \frac{\partial^{2}}{\partial x^{2}}+V(x) .
$$

In one dimension the SWF is given by

$$
\Psi(x)=\phi_{1}(x) \int d x^{\prime} f\left(\left|x-x^{\prime}\right|\right) \phi_{2}\left(x^{\prime}\right) .
$$

The function $\phi_{1}(x)$ is a real-space projection operator (for now we will take it to be one). The term $f\left(\left|x-x^{\prime}\right|\right)$ is chosen $[43,44]$ to be a Gaussian therefore we can write

$$
\Psi(x)=A \int d x^{\prime} \exp \left[-C\left(x-x^{\prime}\right)^{2}\right] \phi_{2}\left(x^{\prime}\right),
$$

where $A$ is the normalization and $C$ is the variational constant.

Let us now start with a wave function of the form

$$
\exp (-\alpha \hat{T})\left|\phi_{2}\right\rangle=\int d x^{\prime} \exp (\alpha \hat{T})\left|x^{\prime}\right\rangle\left\langle x^{\prime} \mid \phi_{2}\right\rangle
$$

in which the kinetic energy propagator is applied to the state $\phi_{2}$. Inserting a momentum identity, and casting the function in the coordinate representation, results in

$$
\begin{aligned}
\left\langle x|\exp (-\alpha \hat{T})| \phi_{2}\right\rangle= & \sqrt{\frac{m}{2 \alpha \pi}} \\
& \times \int d x^{\prime} \exp \left(-\frac{m}{2 \alpha}\left(x-x^{\prime}\right)^{2}\right) \phi_{2}\left(x^{\prime}\right) .
\end{aligned}
$$

The constant $C$ is identified as $C=\frac{m}{2 \alpha}$. The other real-space projection $\phi_{1}(x)$ can be implemented also in the case of a lattice, this would be an example of a Gutzwiller-Baeriswyl projected wave function [4].

\section{E. Implementation}

Before MC sampling the kinetic energy propagator, as well as the estimator, is calculated (Fig. 1) and stored. We apply two types of MC moves. We move the left, central, and right coordinates by standard Metropolis sampling from the distribution $P\left(x_{L}, x_{C}, x_{R}\right)$. We also use exchange moves: two left (or right) particles are randomly chosen and exchanged. These moves are essential for simulating a bosonic system. The calculations below show results from runs on the order of $10^{6} \mathrm{MC}$ steps. The number of independent data points are on the order of $10^{5}$. In our energy calculations error bars typically occurred in the fourth digit of the kinetic or potential energies.

\section{RESULTS}

For a system of $L=100$ sites we calculated the hopping and the potential term. The energy was minimized for different values of $J / U$. The total energy as a function of $J / U$ for 100 and 50 particles based on our variational calculations is shown in Fig. 5. Also shown are results for the same quantity from mean-field theory. As is well known, the mean-field theory of the Bose-Hubbard model [19] gives equations in which the chemical potential is held fixed and the particles fluctuate. We solve the usual mean-field equations for a given $J / U$ adjusting the chemical potential to correspond to an average filling of one and one-half. The figure shows results for the

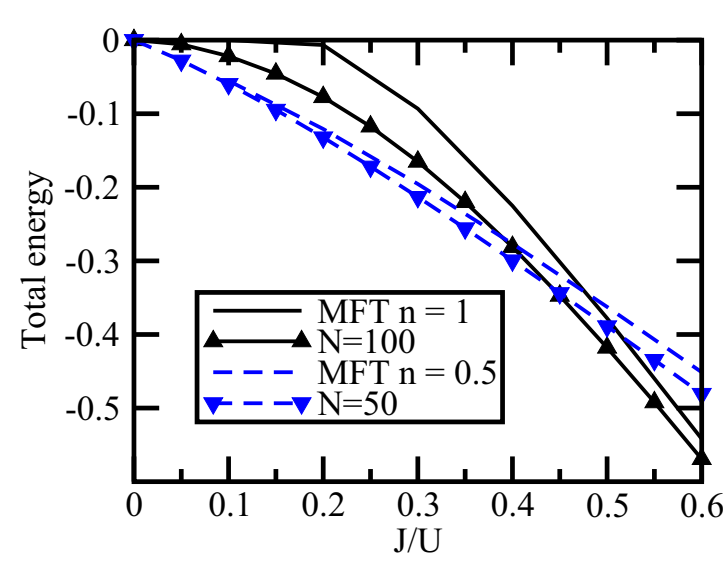

FIG. 5. Energy per lattice site for fillings of one and one-half calculated by our variational Monte Carlo method, and meanfield theory. Lines with symbols represent variational Monte Carlo calculations, lines without symbols are the results of mean-field theory.

total energy without the term proportional to the chemical potential (in order to compare the corresponding quantities from both calculations). The mean-field energies are quite close to the variational Monte Carlo results, but the variational Monte Carlo results are always below the mean-field theory. For small $J / U$ the energy of the system with filling one is larger than the energy for half-filling, but this changes between $J / U=0.4$ and $J / U=0.5$.

The mean-field results indicate a phase transition at fixed filling. At a filling of one the phase transition occurs at $J / U \approx 0.172$, and it can be seen in a discontinuous change in the slope of the energy and the order parameter [19]. In our variational calculations no discontinuity in the slope of the energy is found, although gap closure does occur (discussed below). This result is qualitatively similar to what happens when the BWF is applied to fermions: there also, no metal-insulator transition is found [5] at fixed filling. The curves of the calculated phase diagram (Fig. 6) arise purely as

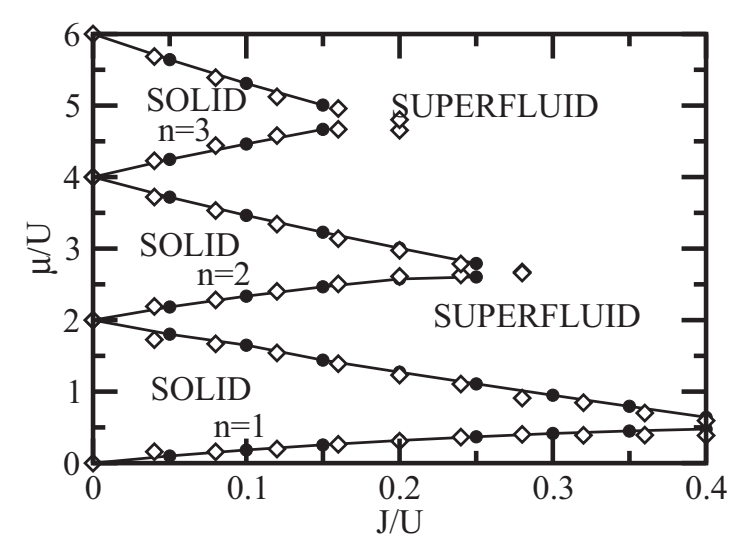

FIG. 6. Phase diagram of the bosonic Hubbard model according to the Baeriswyl wave function (full circles connected by solid line) compared to quantum Monte Carlo results of Rousseau et al. [26] (open diamonds) recalculated via QMC $[35,36] . n$ denotes the filling factor. The error bars in the calculations are smaller than the thickness of the symbols. 
a result of a phase transition which occurs when the particle number is changed; away from integer fillings the phase is superfluid.

To calculate the phase diagram we follow the same procedure, as well as the same parametrization, as Scalettar et al. [25] and Rousseau et al. [26]. Using the definition of the chemical potential $\mu=E(N+1)-E(N)$ we obtain a density vs chemical potential curve. The curve exhibits plateaus at integer fillings (similar to Fig. 2 of Ref. [25]). From the edges of the plateaus the phase diagram can be constructed. The results are shown in Fig. 6. In spite of being a variational method, the results are in good quantitative agreement with the quantum Monte Carlo simulations (cf. Fig. 11 in Ref. [26]). Also, for larger values of $J$ than shown in the figure the gap closes indicating a superfluid phase.

At the tip of the Mott lobe, at integer filling when $J / U$ is varied, a transition is known to occur. From scaling theory it is known to belong to the Kosterlitz-Thouless universality class [19]. The point at which this phase transition occurs can be estimated from inspecting the gap (it closes at the transition point), but since small errors make a big difference at the tip, it can also be obtained [29] from the expression for the gap

$$
\Delta(J)=A \exp \left(-\frac{B}{\sqrt{J_{\mathrm{KT}}-J}}\right) .
$$

Our results also indicate gap closure. For a system with $L=$ 200 lattice sites we obtain $J_{\mathrm{KT}}=0.4604$ (2) from fitting this function to our data for $J>2$, and $J_{\mathrm{KT}} \approx 0.46$ by calculating the point where the gap closes.

The estimates given by our method significantly underestimate the Kosterlitz-Thouless point $J_{\mathrm{KT}}$ compared to other results in the literature [45]. DMRG calculations of Kühner et al. [29] find $J_{\mathrm{KT}}=0.594(2)$, those of Ejima et al. [30] find $J_{\mathrm{KT}}=0.610(2)$, Zakrzewski and Delande [31] find $J_{\mathrm{KT}}=0.5950 \pm 0.020$ for the first Mott lobe, and $J_{\mathrm{KT}}=$ $0.350 \pm 0.004$ for the second one. An exact diagonalization study of Kashurnikov and Svistunov [34] gives $J_{\mathrm{KT}}=$ $0.608(4)$, QMC studies find $J_{\mathrm{KT}}=0.600 \pm 0.010$ [27], and $J_{\mathrm{KT}}=0.610(8)$ [28]. We attribute the discrepancy between the above results and ours to the limitation of the BWF in describing the behavior of the system as $J$ increases. By construction, the BWF is expected $[3,4]$ to produce reliable results for small hoppings.

We also calculated the RDM for several cases. Figure 7 shows the results of our calculations for a system of $L=100$ at filling one for different values of $J / U$. The functions show decay, although there is some deviation from the expected exponential decay (exponential decay implies the absence of a condensate). Our estimates for the correlation lengths $\xi$ for the different cases are: $\xi=0.605(5), 0.95(3), 1.31(5), 1.66(8)$, for $J / U=0.1,0.2,0.3,0.4$, respectively. These results were obtained from fitting a simple exponential function to the calculated RDMs. We emphasize that the exponential functions fit our correlation functions significantly better than power-law decay, as expected.

We have also calculated the RDM for systems away from integer fillings. We used a system of size $L=50$, with particle numbers $N=49$ and $N=48$. In this case, the decay does not reach zero, in other words, a finite condensate fraction is found,

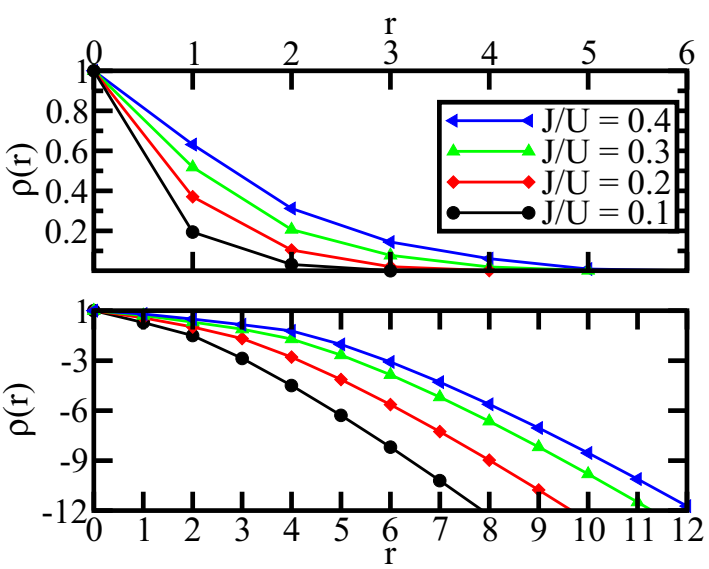

FIG. 7. One-particle reduced density matrix for systems at filling one with $J / U=0.1,0.2,0.3,0.4$. The bottom panel is a semilog plot. The bottom panel is a semilog plot, the $y$ axis is labeled according to powers of ten. The decay of the one-body reduced density matrices is nearly exponential, implying the absence of a condensate.

which is unexpected in one dimension. We emphasize that our variational approach has certain limitations which are likely the cause of this behavior. On one hand, our $U=\infty$ function is represented in a purely combinatorial manner, neglecting correlations between holes or extra particles when near integer filling. This approximation is correct in infinite dimensions. Apart from this, as in the original shadow wave function, a spatially dependent (Gutzwiller) projector could be added to act on the central coordinate, an approach which would improve how correlations are captured. This would correspond to the so-called Baeriswyl-Gutzwiller wave function.

\section{CONCLUSION}

We developed a variational Monte Carlo method for strongly correlated bosonic systems based on the Baeriswyl wave function. Our method was applied to the simple bosonic Hubbard model in one dimension, but it can be generalized to more complex models (e.g., long-range interaction, disorder), and can be applied in any number of dimensions. We calculated the phase diagram of the Bose-Hubbard model, and found excellent agreement with results from quantum Monte Carlo simulations. Our calculations recover the shape of the Mott lobes well. The tip of the Mott lobes is underestimated. We also calculated the one-particle reduced density matrix. At a filling of one we see decay which is nearly exponential.

\section{ACKNOWLEDGMENTS}

We thank Nandini Trivedi and Markus Holzmann for reading our manuscript, and making very useful comments. We are grateful to V. G. Rousseau for providing the results of the stochastic Green's function calculation in Fig. 6. We also thank V. G. Rousseau for very useful discussions. We acknowledge financial support from the Scientific and Technological Research Council of Turkey (TUBITAK) under Grants No. 113F334 and No. 112T176. B.T. acknowledges support from the Turkish Academy of Sciences (TUBA). 
[1] M. C. Gutzwiller, Phys. Rev. Lett. 10, 159 (1963).

[2] M. C. Gutzwiller, Phys. Rev. 137, A1726 (1965).

[3] D. Baeriswyl in Nonlinearity in Condensed Matter, edited by A. R. Bishop, D. K. Campbell, D. Kumar, and S. E. Trullinger (Springer, Berlin, 1986).

[4] D. Baeriswyl, Found. Phys. 30, 2033 (2000).

[5] M. Dzierzawa, D. Baeriswyl, and L. M. Martelo, Helv. Phys. Acta 70, 124 (1997).

[6] W. Metzner and D. Vollhardt, Phys. Rev. Lett. 59, 121 (1987).

[7] W. Metzner and D. Vollhardt, Phys. Rev. Lett. 62, 324 (1989).

[8] W. Metzner and D. Vollhardt, Phys. Rev. B 37, 7382 (1988).

[9] W. Metzner and D. Vollhardt, Helv. Phys. Acta 63, 364 (1990).

[10] H. Yokoyama and H. Shiba, J. Phys. Soc. Jpn. 56, 1490 (1987).

[11] H. Yokoyama and H. Shiba, J. Phys. Soc. Jpn. 56, 3582 (1987).

[12] D. S. Rokhsar and B. G. Kotliar, Phys. Rev. B 44, 10328 (1991).

[13] B. Hetényi, Phys. Rev. B 82, 115104 (2010).

[14] B. Dóra, M. Haque, F. Pollmann, and B. Hetényi, Phys. Rev. B 93, 115124 (2016).

[15] M. A. R. Patoary, S. Chandra, and Y. Kakehashi, J. Phys. Soc. Jpn. 82, 013701 (2013).

[16] M. A. R. Patoary and Y. Kakehashi, J. Phys. Soc. Jpn. 82, 084710 (2013).

[17] Y. Kakehashi, S. Chandra, D. Rowlands, and M. A. R. Patoary, Mod. Phys. Lett. 28, 1430007 (2014).

[18] H. Gersch and G. Knollmann, Phys. Rev. 129, 959 (1963).

[19] M. P. A. Fisher, P. B. Weichman, G. Grinstein, and D. S. Fisher, Phys. Rev. B 40, 546 (1989).

[20] D. Jaksch, C. Bruder, J. I. Cirac, C. W. Gardiner, and P. Zoller, Phys. Rev. Lett. 81, 3108 (1998).

[21] M. Greiner, O. Mandel, T. Esslinger, T. W. Hänsch, I. Bloch, Nature (London) 415, 39 (2002).

[22] J. K. Freericks and H. Monien, Phys. Rev. B 53, 2691 (1996).

[23] G. G. Batrouni, R. T. Scalettar, and G. T. Zimányi, Phys. Rev. Lett. 65, 1765 (1990).

[24] W. Krauth and N. Trivedi, Europhys. Lett. 14, 627 (1991).
[25] R. T. Scalettar, G. Batrouni, P. J. H. Denteneer, F. Hébert, A. Muramatsu, M. Rigol, V. G. Rousseau and M. Troyer, J. Low Temp. Phys. 140, 313 (2005).

[26] V. G. Rousseau, D. P. Arovas, M. Rigol, F. Hébert, G. G. Batrouni, and R. T. Scalettar, Phys. Rev. B 73, 174516 (2006).

[27] V. A. Kashurnikov, A. V. Kravasin, and B. V. Svistunov, JETP Lett. 64, 99 (1996).

[28] S. M. A. Rombouts, K. Van Houcke, and L. Pollet, Phys. Rev. Lett. 96, 180603 (2006).

[29] T. D. Kühner, S. R. White, and H. Monien, Phys. Rev. B 61, 12474 (2000).

[30] S. Ejima, H. Fehske, and F. Gebhard, Europhys. Lett. 93, 30002 (2011).

[31] J. Zakrzewski and D. Delande, AIP Conf. Proc. 1076, 292 (2008).

[32] J. Carrasquilla, S. R. Manmana, and M. Rigol, Phys. Rev. A 87, 043606 (2013).

[33] T. D. Kühner and H. Monien, Phys. Rev. B 58, R14741(R) (1998).

[34] V. A. Kashurnikov and B. V. Svistunov, Phys. Rev. B 53, 11776 (1996).

[35] V. G. Rousseau, Phys. Rev. E 77, 056705 (2008).

[36] V. G. Rousseau, Phys. Rev. E 78, 056707 (2008).

[37] L. Pollet, Rep. Prog. Phys. 75, 094501 (2012).

[38] D. M. Ceperley, Rev. Mod. Phys. 67, 279 (1995).

[39] S. Baroni and S. Moroni, Phys. Rev. Lett. 82, 4745 (1999).

[40] B. Hetényi, E. Rabani, and B. J. Berne, J. Chem. Phys. 110, 6143 (1999).

[41] A. Sarsa, K. E. Schmidt, and R. W. Magro, J. Chem. Phys. 113, 1366 (1999).

[42] O. Penrose and L. Onsager, Phys. Rev. 104, 576 (1956).

[43] L. Reatto and G. L. Masserini, Phys. Rev. B 38, 4516 (1988)

[44] S. Vitiello, K. Runge, and M. H. Kalos, Phys. Rev. Lett. 60, 1970 (1988).

[45] The results reported here for comparison are based on a different definition of the Bose-Hubbard model, in which the potential is divided by two. For this reason, the results reported in the original papers are half of the values cited here. 